\title{
RETRACTED ARTICLE: Slowly Modulated Two-Pulse Solutions in a Generalized Klausmeier-Gray-Scott Model
}

\author{
Xijun Deng1 ${ }^{1}$ Shangjiang Guo ${ }^{1}$
}

Received: 22 October 2015 / Accepted: 21 January 2016 / Published online: 4 February 2016

(c) Malaysian Mathematical Sciences Society and Universiti Sains Malaysia 2016

This article has been retracted by the Editor-in-Chief in agreement with the authors because it contains portions of text that apparently have been duplicated from "Pattern formation in a generalized Klausmeier-Gray-Scott model" by Lotte Sewalt, www. math.leidenuniv.nl/scripties/MasterSewalt.pdf.

Electronic supplementary material The online version of this article (doi:10.1007/s40840-016-0303-9) contains supplementary material, which is available to authorized users.

$凶$ Xijun Deng

xijundeng@aliyun.com

1 College of Mathematics and Econometrics, Hunan University, Changsha 410082,

People's Republic of China 